\title{
The Research and Design of Remote MCU Learing Plaform Based on the Combination of Virtual and Reality Chunrong CHEN $^{1, a}$, Kaijin QIU ${ }^{1, b}$, *
}

${ }^{1}$ College of Computer \& Information Science Southwest University, Chongqing, 400715, China

aemail: 13638396486@163.com, bemail: qkjqkj@swu.edu.cn, "corresponding author

Keywords: Remote Learning, MCU learning, Virtual and Reality combination

\begin{abstract}
Recently, there have been a great need in society for MCU learning especially in engineering and science. However, the environment development didn't match the increasing demand for MCU teaching in fact. On the one hand, the costs of learning at their own expense are high. On the other hand, limits of learning conditions and shortage of devices and space in MCU laboratories still exist. In this circumstance, the paper designs a remote MCU learning platform based on virtual reality where the MCU learners can learn MCU programming through network instead of on the MCU. The platform with low costs in this paper is not only convenient and user-friendly, but also reliable.
\end{abstract}

\section{Introduction}

MCU technology has come a long way in the past 40 years since it appeared. It has been widely used in the fields such as automobile industry, medicine, commercial offices, as well as various kinds of intelligent instruments like mobile phone and so on[1]. In addition, there is no doubt that it has penetrated into every aspect of our lives. With the development of MCU technology and MCU learning, MCU has become a necessary skill for the learner who studies things about science technology.

At present, there are mainly two types of MCU programming learning for students at college:

In the first type, Users buy a MCU with off-the-shelf peripheral equipment, including keyboard, LCD, external interrupt, I2C, SPI, PWM module and so on at the price of hundreds RMB. It brings following disadvantages. First, it is expensive. Second, it is inconvenient to be repaired when broken as most learners are unable to repair it on their own. Third, it is also not easy to carry with them when using it because of the peripheral equipment.

The other type doesn't need to buy a MCU, instead, they simulate on the software like Keil, proteus and so on after programming, which brings the disadvantage that the result is not always correct because of the differences between the real circuit and simulation software.

To solve the problems above, this paper designs a platform of long-distance MCU learning based on virtual reality which has lower costs and is more convenient. Above all, it can get a correct result at the same time. It has following advantages:

\section{Compared with traditional MCU}

Low costs: The platform of long-distance MCU learning based on virtual reality is built by an embedded and extensible way which is highly integrated and is convenient to repair, and it is easy for the administrator to manage. What's more, the platform can save the costs on devices for the users only need to program on the PC.

Convenience: The platform allows the users to program and simulate at any place whenever it is which makes the platform more user-friendly and convenient.

\section{Compared with simulation software}

The platform in this paper solves the problems like that the result is incorrect sometimes and the lack of peripheral equipment compared with simulation software. Moreover, the platform only needs users to setup the app or a software which needs small memory footprint, making the simulation runs faster [2].

The platform adopts one-to-many mode, which has following features: First, the MCU system 
will be occupied only when a user downloads his program to the MCU. It means that the MCU will not be occupied when programming. Second, the terminal works 24 hours a day and the system can expand and change the number of the MCU terminals depending on the condition and the demands so that the system can work efficiently.

As is illustrated above, the platform of long-distance MCU learning based on virtual reality is of great integration and modularity. It is fixed in laboratories so that its operation life can be longer and it can be repaired immediately if it breaks down. Last but not least, less administrators is needed, decreasing the costs.

\section{Overall design of the remote MCU learning platform}

Architecture of the platform. As is illustrated in Fig.1, The Learning platform is composed of the client which includes smart phone terminal and computer terminal, cloud platform server terminal and MCU platform system connected by Network router. The remote MCU learning platform adopts $\mathrm{C} / \mathrm{S}$ structure which means Client/Server [3]. Center processor transfers information between learner and MCU terminal by connecting all ports. When the learner asks for program simulation through the smart phone terminal or the computer terminal, the client carries on the effective response, and transmits the request to the cloud platform server through the network .Then the server transfers the request to network router of laboratory through the network and matches the idle MCU system. MCU system runs the corresponding program based on the learner's request, and return the simulation results to the client. After that, it becomes possible for the learner to browse the results intuitively.

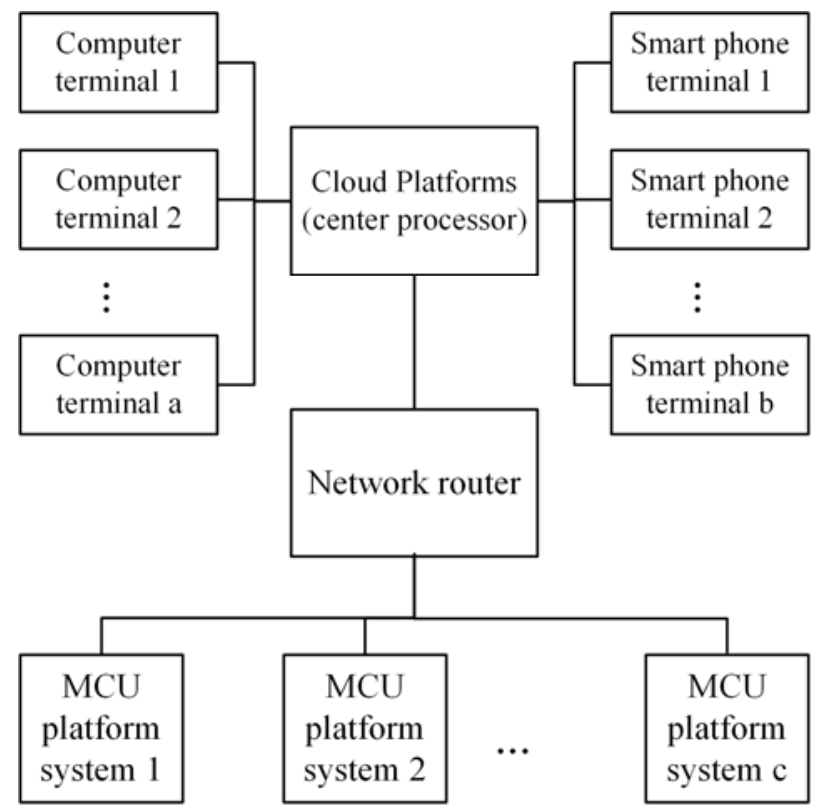

Fig. 1. System Structure

Client. The client of the remote MCU learning platform includes smart phone terminal and computer terminal, it provides learning environment to MCU learner and shows the experimental results. The client creates virtual LCD, keyboard and other peripheral equipment for easily interacting with students. The students get the result of experimental data through cloud platform after that they log on client software on the local area network (LAN) or mobile phone app and choose to program or simulate. The client allows students to program or simulate no matter when it is and where you are.

Server. As the core of the remote system, server is a bridge that connects the user with experimental equipment remotely [4].

It adopts Ali cloud platform to verify the user log in, save the user information and read the data sent from one of host computer and host computer and then send the data to the other one after processing and allocating the MCU resource for users. 
MCU platform system. MCU platform system controls microcontroller to download and run the program. In addition, it returns the experiment data to the platform.

\section{Design of the MCU platform system}

As is illustrated in Fig.2, The MCU platform system is composed of FPGA gathering module, Downloader, micro-controller operation module, Voltage and Current sampler and embedded host.

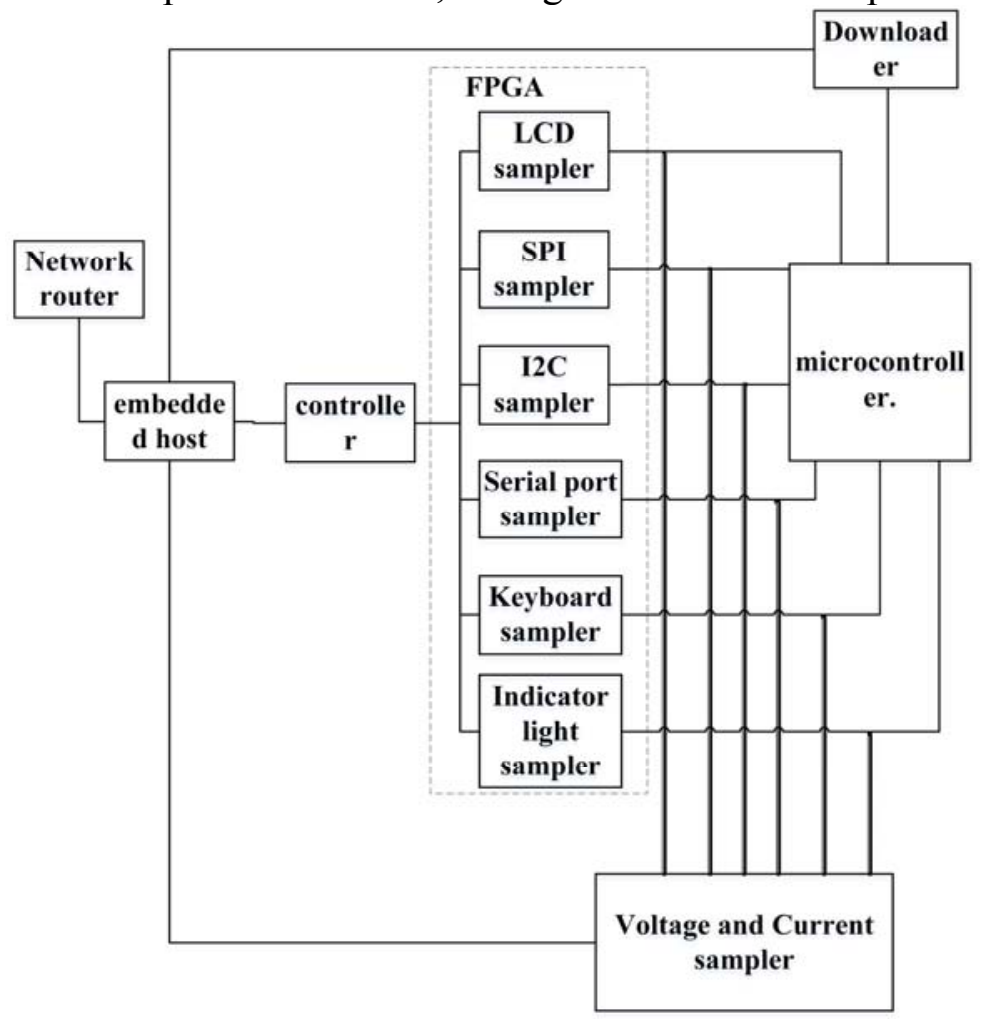

Fig. 2. A MCU platform system structure

FPGA gathering. The MCU adopts FPGA to send the parameters from peripheral equipment to the network server. FPGA collects data from LCD, SPI, I2C, serial port, keyboard, indicating lamp automatically and then sends it to the controller for further processing. The entire process of processing can be divided into the following four parts: sampling, maintaining, quantization and coding. First, different analog signals input is sampled and then they stay the same for some time, during which electric parameters are turned into digital values and coded by the controller. Finally, the conversion result according to certain coding format is showed. Here the first period finished and next period started [5].

Controller. The electrical parameter collected from LCD sampler, SPI sampler, I2C sampler, serial ports sampler, keyboard sampler and indicator light becomes intuitive electric parameter after processed by controller. All data or results including graphics are transmitted to the user terminal in the form of text through the cloud platform after processing for less cost, higher efficiency and shorter time of information transmission.

Voltage and Current sampler. It captures the voltage and current signals from all the pins of the MCU. On the one side, the voltage signals after filtration from divider resistance are captured periodically by the MCU and turned into digital values. On the other side, current signals output from the current diverter are multiplied and filtrated after captured and are turned into digital values to show the port voltage and port current to the MCU learner.

Downloader. As is illustrated in Fig.3. The downloader adopts USB and UART Protocol. The network router communicates with MCU by serial port and MCU places the program received from downloader in a fixed program memory (on-chip ROM or EPROM) to download program from external downloader and run again. 


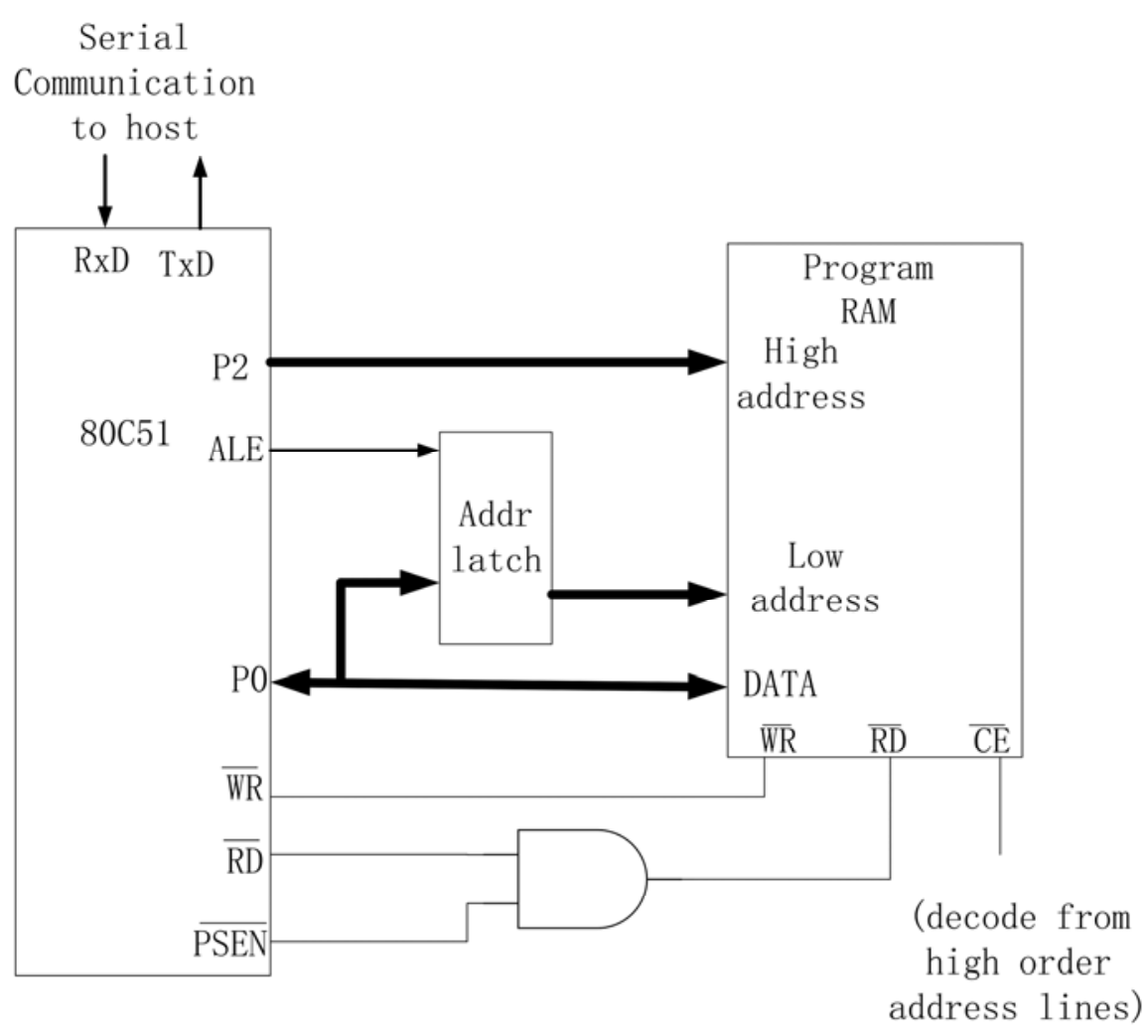

Fig. 3. Link of downloader structure

Microcontroller module. Microcontroller module is the main part of the system terminal who runs the program. It is the hardware platform of the remote laboratory, including a microcontroller and the peripheral equipment and interfaces which are used to communicate with microcontroller directly. The kind and the number of microcontroller could change according to the demands of the users.

Embedded host. The embedded host is supposed to link to the network and work out the assigned addresses of the information input and output. It can download program, upload the simulation text and transmit signal of the electrical parameters of each pin automatically through three different channels to make sure that there is no conflict while sending data to the network server.

\section{Software flow of the platform}

The technologies used in the system include the connection and communication between the PC and the MCU, information uploading of resources inside and outside and long-distance downloading. The flow of the software is illustrated in Figure 4.

Learners program on the PC or smart mobile phone and link to network router. Then the network router downloads the program to the MCU system and MCU system selects an unoccupied microcontroller to execute programs and simulate automatically. At the same time, the selected microcontroller enables the necessary peripherals and interfaces automatically which measure the data related after one of the pins gets the signal. Finally, the host computer shows the result according to the signal, presenting the virtual but real situation of LCD, keyboard and touch screen and getting the waveform based on the signal which reflects the real results of the microcontroller.

The platform is supposed to process the text to be sent before it sends the simulation result to the client. The text data sent by peripheral equipment is showed in table 1. Learner can also put forward special requirements when programming such as transmitting the data like images without processing and then controller transfers the collected signal to the client directly without text processing to make the result easier to understand. 


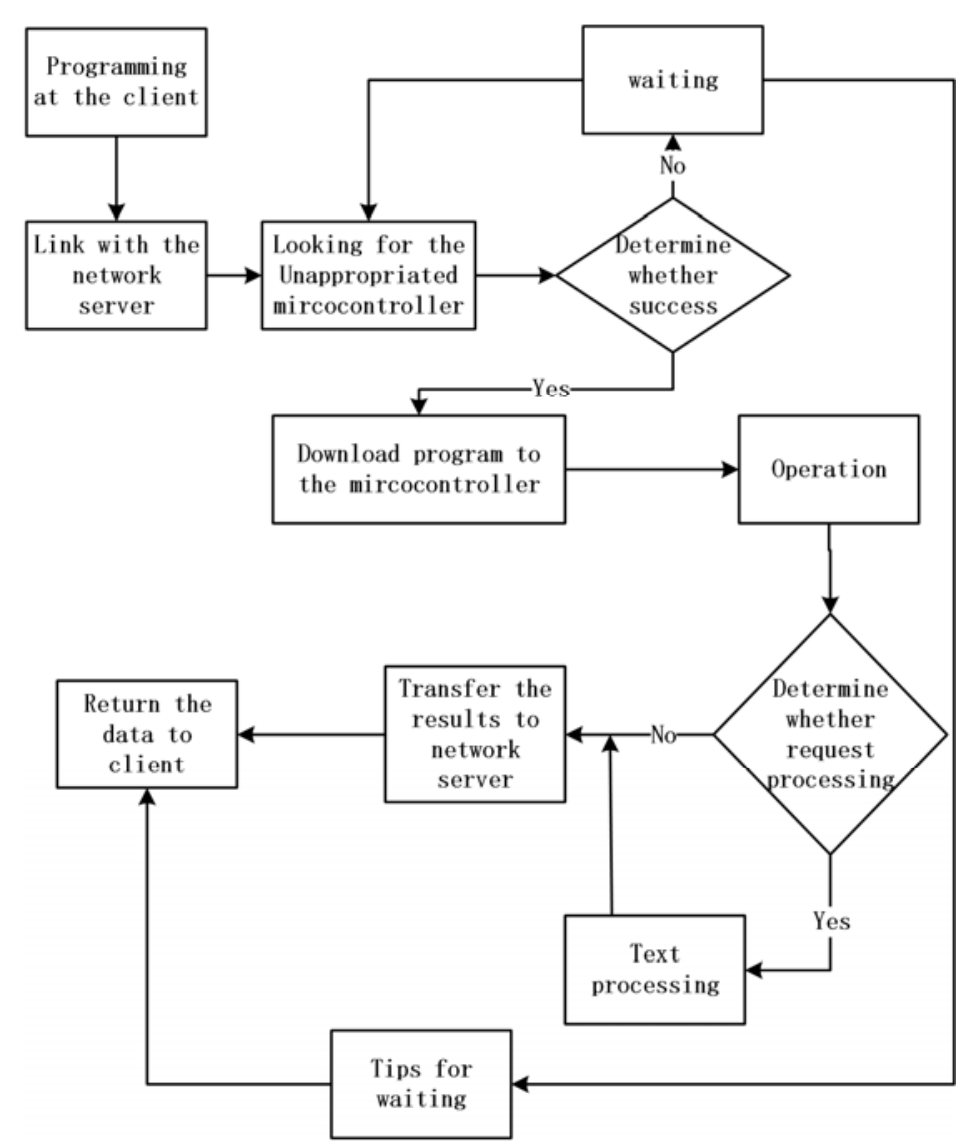

Fig. 4. Software flow

Table1. Transformation content

\begin{tabular}{|c|c|}
\hline Transformation content & text content \\
\hline Basic contents & $\begin{array}{l}\text { Download successfully/ unsuccessfully, The time required to } \\
\text { download, serial number of the working MCU }\end{array}$ \\
\hline LCD & The display text of LCD \\
\hline I2C & The content of $\mathrm{I} 2 \mathrm{C}$, The transmission time of $\mathrm{I} 2 \mathrm{C}$ \\
\hline Keyboard & The press state of keyboard \\
\hline Indicator light & The lamplight of Light \\
\hline Buzzer & Start-stop state of the buzzer, Working time of the buzzer \\
\hline $\mathbf{A D} / \mathbf{D A}$ & Current/voltage data \\
\hline
\end{tabular}

\section{Conclusion}

After entering the information age, it has become more and more important to provide the MCU learners with a convenient, low-costs and flexible learning platform. This paper shows the background of MCU learning firstly and then comes up with the physical structure and the software of the platform of long-distance MCU learning based on virtual reality which allows users to program and simulate by the software on the $\mathrm{PC}$ anywhere no matter what time it is.

\section{Acknowledgement}

This work is partially supported by the Augmented Reality Scene of Blankness-Actuality Content Knowledge Representation and Understanding Fusion Technology (cstc2012ggC40004). 


\section{References}

[1] Shi Zhengyin, Zhu Shan'an, The Research and Design of A Virtual Electrical and Electronic Network Laboratory Based on the Modelica [J], Computer Engineering. 11th issue, 2012.

Zhang huan, Remote Network Experiment of Digital Circuit Under The Framework of Virtual Reality [D], Zhejiang University, 2014.

[3] Li Yunyun, The Analysis of The B/C and C/S Architecture [J], Friend of Science Amateurs, 1st issue, 2011.

[4] Zhang Shouheng, The Research and Design of The Server of Virtual Reality Network Laboratory [D], Zhejiang University, 2013.

[5] Chu Yidong, A Study on Data Acquisition System Based on FPGA [D], Changchun University of Science and Technology, 2009.

[6] Cui Zhangkun, The Research and Design of High-precision Voltage and Current Detection System of Lithium-ion Battery Pack in Electric Cars [D], Shenyang Ligong University, 2012. 